\title{
Helping doctors by helping patients meet social needs
}

\author{
n Cite as: CMAJ 2019 September 23;191:E1064. doi: 10.1503/cmaj.1095805
}

Posted on cmajnews.com on Sept. 4, 2019

H ealth facilities that increase their capacity to address the social needs of patients may also improve the health of their physicians, according to a study on physician burnout in the Journal of the American Board of Family Medicine. conducted by the Canadian Medical Association. This growing problem has been called "a public health crisis" that has "reached global epidemic levels."

There are many contributing factors to physician burnout, including administrative burden, work-related stress and poor

needs, the authors analyzed survey data from the burnout module of certification applications to the American Board of Family Medicine in 2016. Self-reported information was used from 1298 family physicians who practise in ambulatory primary care settings.

Just over a quarter $(27 \%)$ of the doctors reported symptoms of burnout, but "respondents who reported high clinic capacity to address patients' social needs had lower odds of burnout than those reporting lower capacity to address patients' social needs." The physicians in high-capacity clinics were about a third less likely to experience burnout. There was a "modest but statistically significant inverse association" that was "as strong as that between self-reported EHR [electronic health record] proficiency and burnout." The settings most likely to be reported as having a high capacity to meet social needs of patients included a social worker and a pharmacist.

"As upstream interventions, including those focused on legal needs, food and housing security, or social support, become more prevalent in the US health care

Upstream interventions, such as ensuring access to food and shelter, may not only improve patients' health, but could also potentially improve physicians' health.

In the United States, it is estimated that $60 \%$ of physicians have some symptoms of burnout, the paper notes. About a third of Canadian doctors report symptoms of burnout or depression, including emotional exhaustion and depersonalization, according to a 2018 national survey clinical leadership. One factor that hasn't received adequate attention, according to the study authors, is the capacity of a physician's workplace to meet patients' social needs.

To examine the association between burnout and meeting patients' social system, it will be useful to assess not only how those programs influence patient health, cost, and utilization but how they may affect physician burnout and retention," the authors concluded.

Roger Collier, CMAJ 